BMJ Open Sport \& Exercise Medicine

\title{
Systematic assessment of the growth plates of the wrist in young gymnasts: development and validation of the Amsterdam MRI assessment of the Physis (AMPHYS) protocol
}

Laura S Kox, ${ }^{1,2,3}$ Rik B J Kraan, ${ }^{1,2,3}$ Kees F van Dijke, ${ }^{4}$ Robert Hemke, ${ }^{1}$ Sjoerd Jens, ${ }^{1}$ Milko C de Jonge, ${ }^{5}$ Edwin H G Oei, ${ }^{6}$ Frank F Smithuis, ${ }^{1}$ Maaike P Terra, ${ }^{7}$ Mario Maas ${ }^{1,2,3}$

To cite: Kox LS, Kraan RBJ, van Dijke KF, et al. Systematic assessment of the growth plates of the wrist in young gymnasts: development and validation of the Amsterdam MRI assessment of the Physis (AMPHYS) protocol. BMJ Open Sport \& Exercise Medicine 2018:4:e000352. doi:10.1136/ bmjsem-2018-000352

- Additional material is published online only. To view please visit the journal online (http://dx.doi.org/10.1136/ bmjsem-2018-000352)

Accepted 22 February 2018
Check for updates

For numbered affiliations see end of article.

Correspondence to Laura S Kox; I.s.kox@amc.uva.n

\section{ABSTRACT}

Objectives To develop and validate a protocol for MRI assessment of the distal radial and ulnar periphyseal area in gymnasts and non-gymnasts.

Methods Twenty-four gymnasts with wrist pain, 18 asymptomatic gymnasts and 24 non-gymnastic controls (33 girls) underwent MRI of the wrist on a 3T scanner. Sequences included coronal proton densityweighted images with and without fat saturation, and three-dimensional water-selective cartilage scan and T2 Dixon series. Skeletal age was determined using hand radiographs. Three experienced musculoskeletal radiologists established a checklist of possible (peri) physeal abnormalities based on literature and clinical experience. Five other musculoskeletal radiologists and residents evaluated $30 \mathrm{MRI}$ scans (10 from each group) using this checklist and reliability was determined using the intraclass correlation coefficient (ICC) and Fleiss' kappa. A final evaluation protocol was established containing only items with fair to excellent reliability. Results Twenty-seven items were assessed for reliability. Intra-rater and inter-rater agreement was good to excellent (respective ICCs $0.60-0.91$ and $0.60-0.78$ ) for four epiphyseal bone marrow oedema-related items, physeal signal intensity, metaphyseal junction and depth of metaphyseal intrusions. For physeal thickness, thickness compared with proximal physis of first metacarpal, metaphyseal intrusions, physeal connection of intrusions and metaphyseal bone marrow signal intensity, intra-rater agreement was fair to excellent (ICC/kappa 0.55-0.85) and inter-rater agreement was fair (ICC/kappa 0.41-0.59). Twelve items were included in the final protocol.

Conclusion The Amsterdam MRI assessment of the Physis protocol facilitates patient-friendly and reliable assessment of the (peri)physeal area in the radius and ulna.

\section{INTRODUCTION}

In young athletes, physeal injury can occur as traumatic fractures or as stress injuries caused by repetitive microtrauma. ${ }^{1}$ The latter
What are the new findings?

The concise Amsterdam MRI assessment of the Physis (AMPHYS) protocol contains 12 items derived from the literature and clinical experience that can be used to assess characteristics of the periphyseal area in the distal radius and ulna.

- All 12 items of the AMPHYS evaluation protoco showed good content validity and fair to excellent intra-rater and inter-rater agreement.

- The accompanying 15 min MRI protocol includes coronal three-dimensional frequency-selective, fatsuppressed gradient-echo imaging, a T2-weighted two-point Dixon sequence and proton densityweighted sequences with and without fat suppression.

How might it impact on clinical practice in the future?

- The AMPHYS protocol contributes to uniform assessment and reporting of suspected physeal stress injury on MRI, with minimal patient and clinician burden.

- Use of the AMPHYS protocol in research and clinical practice provides more insight in morphological differences between stress-induced and maturityrelated changes in the periphyseal area.

are commonly located at physes of the distal radius (gymnast wrist), the distal humerus (Little League elbow) and the proximal humerus (Little League shoulder). ${ }^{2}$ In the physis, or growth plate, cartilage cells are generated, proliferate, hypertrophy, and eventually calcify into bone, ${ }^{3}$ vascularised by metaphyseal and epiphyseal vessels. ${ }^{4}$ Injury to the multilayered physis or its vascularisation can cause (partial) physeal growth arrest, 
sometimes resulting in permanent growth disturbances and damage to surrounding structures. ${ }^{5}$

Wrist pain and overuse wrist injury occur frequently in young gymnasts, ${ }^{6}$ and early diagnosis of physeal stressinjury allows timely intervention and recovery to prevent longterm sequelae. ${ }^{7}$ Radiographic signs include distal radial physeal irregularity and widening. ${ }^{8}$ These characteristics are incorporated in a radiographic grading system, irregularity representing grade 1 injury and widening severe (grade 3) injury. ${ }^{9}$ MRI can, in addition, depict non-osseous tissues like physeal cartilage, the highly vascular primary metaphyseal spongiosa and traumatic signs like bone marrow oedema (BMO). ${ }^{40}$ Cartilage-sensitive and fluid-sensitive MRI sequences such as three-dimensional (3D) frequency-selective, fat-suppressed gradient-echo images and fat-saturated T2-weighted images have been recommended for detailed imaging of physeal cartilage and stress-induced BMO. ${ }^{71011}$ Dixon chemical shift MRI can be used to achieve uniform fat suppression in the hand and wrist. ${ }^{12}$

MRI has been proposed as the imaging method of choice for evaluation and therapeutic decision-making in patients with physeal stress injury. ${ }^{13}$ However, some injury manifestations may resemble normal growth and physeal development on MRI, ${ }^{4}$ and BMO is often present in wrists of asymptomatic children. ${ }^{14} 15$ This renders uniform assessment and diagnosis of physeal stress injury challenging, especially at an early stage. In addition, the number of potential injury signs described on radiographs, and translated to MRI, is extensive and may require lengthy MRI examinations and evaluations. A concise and reliable procedure for MRI-based assessment of the physis can aid in identifying physeal injury in a patient-friendly manner. This study aimed to develop and validate a standardised protocol for MRI evaluation of the distal radial and ulnar periphyseal area to improve uniform assessment of the wrist growth plates.

\section{METHODS}

\section{Study design}

This study was performed according to the Declaration of Helsinki and approved by our institution's review board (reference no 2014_382\#B2015303). It consisted of multiple phases: prospective MRI collection, literature-based and expert-based protocol development, and reliability testing by different experts. MRI acquisition took place at the Academic Medical Center, Amsterdam, between June 2015 and November 2017. Participants and their parent(s) or guardian(s) provided written informed consent to participate.

\section{Study population}

The study cohort consisted of 24 gymnasts with wrist pain, 18 asymptomatic gymnasts and 24 non-gymnasts, aged $12-18$ years. Symptomatic gymnasts were referred by their (sports) physician. Sex-matched and skeletal age-matched asymptomatic gymnasts and non-gymnasts were recruited through gymnastics clubs, the bring-a-friend strategy and notices within our institution. Gymnasts had performed their sport for at least 1 year and up to 6 months or less prior to participation. The symptomatic group consisted of gymnasts with clinically suspected physeal injury and wrist pain in the 6 months prior to inclusion. The non-gymnast group consisted of healthy children without wrist pain. Exclusion criteria were the following: history of fracture, wrist surgery or infection, growth disorders, systemic or oncological disease involving the musculoskeletal system (eg, juvenile idiopathic arthritis) and closed growth plate on hand radiograph. Participants filled out a questionnaire on demographic information, sports participation and wrist pain.

\section{Imaging}

Standard posterior-anterior radiographs with focusdetector distance of $1.30 \mathrm{~m}$ were obtained of one (symptomatic) hand and wrist. Skeletal age was determined using a computerised method (BoneXpert, v1.0; Visiana, Holte, Denmark; www.BoneXpert.com) validated in a healthy Dutch population. ${ }^{16}$ MRI of the (symptomatic) wrist was performed in a feet-first, supine position with both arms resting alongside the body on a 3.0 Tesla MRI scanner (Ingenia; Philips Healthcare, Best, The Netherlands) using a dedicated eight-channel, receive-only wrist coil.

The MRI protocol included coronal turbo spin-echo (TSE) proton density (PD)-weighted sequences with and without fat suppression (spectral attenuated inversion recovery) as part of our institution's standard clinical protocol. Two additional sequences were used: a coronal T2-weighted two-point Dixon sequence and a coronal 3D water-selective cartilage scan (3D WATSc) (online supplementary appendix 1). The field of view was centred on the distal radial and ulnar physes and included the proximal physis of the first metacarpal bone (MC-1) on all images (online supplementary appendix 1).

\section{Development of Amsterdam MRI assessment of the Physis (AMPHYS) protocol}

Following guidelines for achieving good content validity, ${ }^{17}$ potentially relevant growth plate characteristics and physeal (stress) injury signs were collected from the literature (figure 1). ${ }^{17}$ The expert group in the development phase consisted of three experienced musculoskeletal radiologists (MCdJ, EHGO, MM) from different institutions and two physicians experienced in research on musculoskeletal imaging (LSK, RBJK). The senior author added items to the literature-derived list, based on the experience in evaluating physeal stress injuries on standard clinical MRI. A standardised scoring form with these items was created, allowing radiologists to indicate a finding's presence in any image series, its extent or severity, its location in the radius and/or ulna, and which sequences provided optimal assessability.

After the introduction of the sequences comprising the MRI protocol, the radiologists individually evaluated a random sample of blinded MRI scans from each of the 


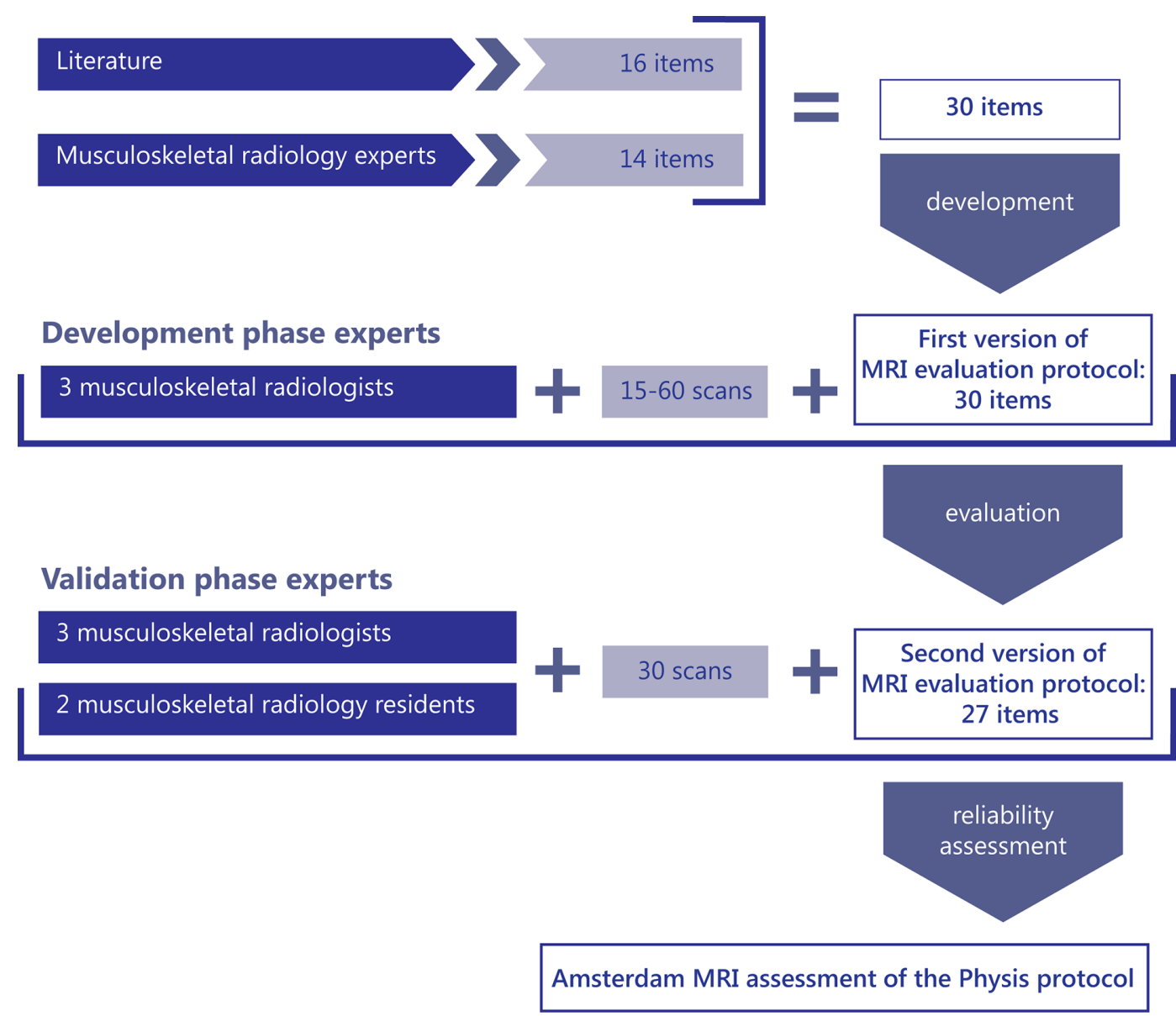

Figure 1 Flow chart showing the development process of the Amsterdam MRI assessment of the Physis evaluation protocol.

participant groups. Image evaluation was performed on a PC workstation with high-resolution monitor using IMPAX software version 6.6.1.4024 (AGFA HealthCare N.V., Mortsel, Belgium). One radiologist (MM) assessed scans of 60 participants, the others (MCdJ, EHGO) of at least 15 participants. The expert group evaluated the reading directly afterwards, discussing disagreements in scoring until consensus was reached. Items considered not relevant or not assessable on MRI were removed. An initial MRI scoring checklist was formed, including instructions and images illustrating abnormalities.

\section{Validation of AMPHYS protocol}

The expert group in the validation phase was not involved in protocol development and consisted of three musculoskeletal radiologists and two musculoskeletal radiology residents (KFvD, FFS, MPT, SJ, RH) from three institutions, representing daily radiological practice. To assure consensus on interpretation of scoring instructions, the group discussed the newly developed MRI scoring checklist and examples, and assessed multiple trial cases prior to image evaluation. Using the same workstations and software as the development group, all raters evaluated 30 blinded MRI scans, that is, 10 from each participant group (figure 1). Four weeks after the first session, one rater $(\mathrm{RH})$ re-evaluated all 30 MRI scans to determine intra-rater agreement.

\section{Statistical analysis}

Inter-rater agreement of scoring items was determined by calculating intraclass correlation coefficients (ICCs) for absolute agreement between raters using a two-way random analysis of variance (ANOVA) model (case 2, $\operatorname{ICC}(2,1))$ for ordinal variables, and the unweighted Fleiss' kappa for binary variables. The set of 30 double assessments by one rater was used to calculate the intrarater ICC for absolute agreement for each item, using a two-way random ANOVA model (case 2, ICC $(2,1)$ ) for ordinal variables, and the unweighted Fleiss' kappa for dichotomous variables. Agreement was expressed by the ICC or kappa and its CI, and levels of agreement measured by ICC were defined according to Cicchetti ( $<0.4$, poor; 0.4 to 0.59 , fair; 0.6 to 0.74 , good; $\geq 0.75$, excellent), and for kappa, according to Landis and Koch ( $<0$, poor; 0 to 0.2 , slight; 0.21 to 0.4 , fair; 0.41 to 0.6 , moderate; 0.61 to 0.8 , substantial; 0.81 to 1.0 , almost perfect). ${ }^{18} 19$ Only items with fair to excellent inter-rater and intra-rater agreement were considered to show sufficient agreement and variability within the population and included in the final protocol. Based on an expected ICC $\geq 0.8$ and preferred $95 \%$ CI of 0.6 to 1.0 , the preferred sample size was eight images per group to be rated by each rater. Data analyses were performed using SPSS V24.0. 


\section{RESULTS}

\section{Participant characteristics}

The cohort consisted of 33 boys and 33 girls. MRI scans for inter-rater and intra-rater agreement were of five girls and five boys in the symptomatic gymnast group, six girls and four boys in the asymptomatic gymnast group and seven girls and three boys in the non-gymnast group. Respective mean calendar and skeletal ages were 14.7 (SD 1.3) and 13.0 (SD 0.7) years in symptomatic gymnasts, 14.1 (SD 1.0) and 12.2 (SD 1.0) years in asymptomatic gymnasts and 13.5 (SD 1.1) and 13.0 (SD 1.8) years in non-gymnasts.

\section{Content validity}

The development phase rendered 16 relevant items from the literature and 14 items from clinical experience. After the first reading and consensus meeting, three items were excluded (physeal depressions, metaphyseal cysts ${ }^{20}$ and striations ${ }^{9}$ ). The remaining 27 items were considered relevant and dichotomous or ordinal variables assessable on MRI. Items were divided into three categories: epiphysis (ie, various characteristics of BMO), physis (ie, thickness, signal intensity, disruptions and epiphyseal border) and metaphysis (ie, physeal border, intrusions, $\mathrm{BMO}$, widening, periosteal bone formation and sclerosis) (table 1). Preferred sequences were defined per item and visibility of BMO on the cartilage-specific 3D WATSc sequence was identified separately to potentially identify very severe BMO. Figure 2 shows the normal multilayered aspect of the physis, and figure 3 shows examples of epiphyseal BMO, physeal thickening, metaphyseal intrusions and metaphyseal BMO.

\section{Inter-rater agreement}

In the radius, inter-rater agreement was excellent for BMO extent and signal intensity (ICC, 0.78 and 0.76 , respectively) (table 2). For BMO location and visibility in the epiphysis on the 3D WATSc sequence, inter-rater agreement was good (ICC 0.60 and 0.70 , respectively), as well as for physeal signal intensity (ICC 0.62), metaphyseal border of physis (ICC 0.60) and depth of intrusions into the metaphysis (ICC 0.69). Agreement was moderate for metaphyseal intrusions (kappa 0.59) and physeal thickness (kappa 0.47), and fair for physeal thickness compared with the proximal MC-1 physis (ICC 0.58), physeal connection of metaphyseal intrusions (ICC 0.41) presence (kappa 0.32) and signal intensity of metaphyseal BMO (ICC 0.51), physeal border on epiphyseal side (kappa 0.35) and periosteal bone formation (kappa $0.26)$.

In the ulna, inter-rater agreement was moderate for metaphyseal intrusions (kappa 0.57). Agreement was fair for metaphyseal border of the physis (ICC 0.43), connection of metaphyseal intrusions with physis (ICC 0.43 ), intrusion depth (ICC 0.54), presence (kappa 0.33) and signal intensity of metaphyseal BMO (ICC 0.43) and physeal thickness (kappa 0.22). Agreement was poor or slight for the other items.

\section{Intra-rater agreement}

For the radius, intra-rater agreement was excellent or substantial for extent, signal intensity and visibility of epiphyseal BMO (ICC 0.86, 0.85 and 0.90 , respectively), as well as for physeal thickness (kappa 0.80), thickness compared with the proximal MC-1 physis (ICC 0.83), signal intensity (ICC 0.81), metaphyseal border (ICC 0.84), metaphyseal intrusions (kappa, 0.85) and their physeal connection (ICC 0.85) and depth (ICC 0.91) (table 2). Agreement was good for epiphyseal BMO location (ICC 0.60) and epiphyseal border of physis (ICC $0.67)$, and fair for metaphyseal BMO signal intensity (ICC 0.55 ) and visibility on 3D WATSc (ICC 0.43 ).

Intra-rater agreement for ulnar items was good or substantial for extent (ICC 0.74) and location (ICC 0.69) of epiphyseal BMO, physeal signal intensity (ICC 0.62), and metaphyseal intrusion presence (kappa 0.71) and depth (ICC 0.67). Agreement was moderate or fair for epiphyseal BMO signal intensity (ICC 0.59), metaphyseal BMO (ICC 0.46), physeal thickness (kappa 0.44), thickness compared with the proximal MC-1 physis (ICC 0.52), metaphyseal border (ICC 0.58 ) and intrusion connection with the physis (ICC 0.56). The remaining items showed poor or slight agreement.

The final AMPHYS protocol consisted of 12 items for the radius and 5 for the ulna (table 3 , online supplementary appendix 2) and a scoring form (online supplementary appendix 3).

\section{DISCUSSION}

The AMPHYS protocol contains 12 elements with good content validity to assess characteristics of the physis, epiphysis and metaphysis of the radius and ulna, with fair to excellent inter-rater and intra-rater agreement.

\section{Bone marrow oedema}

While BMO can indicate injury, it can be present in wrist bones of $40 \%-49 \%$ of asymptomatic children, especially during rapid skeletal maturation. ${ }^{1415}$ Local areas of focal periphyseal oedema (FOPE) have been described as early sign of physiological physeal fusion. ${ }^{21}$ In young athletes, asymptomatic BMO may result from a physiological stress response to exercise. ${ }^{22}{ }^{23}$ We therefore expected that periphyseal BMO would be present in all three groups, but its characteristics might be used to differentiate between physiological and injury-related BMO.

While five BMO-related items in the epiphysis and metaphysis showed fair to excellent intra-rater and inter-rater agreement, reliability was best for multiple characteristics of epiphyseal BMO (figure 3A). Periphyseal BMO was present in all groups, but its signal intensity and epiphyseal extent and location showed fair to excellent agreement for the ulna and the radius. Gradient-echo sequences commonly used for (physeal) cartilage imaging, such as 3D WATSc, are well-known to be insensitive for BMO, compared with fat-suppressed, T2-weighted fast spin-echo sequences. ${ }^{24}$ However, our 
Table 1 Scoring items and grades for physeal characteristics in the distal radius and ulna

\section{Scoring item}

Grades

Epiphysis

Bone marrow oedema

\begin{tabular}{|c|c|c|c|c|}
\hline Extent ${ }^{\star}$ & No oedema & \multicolumn{2}{|c|}{$<50 \%$ of epiphyseal volume } & $>50 \%$ of epiphyseal volume \\
\hline Location $^{1032}$ & No oedema & \multicolumn{2}{|c|}{ Oedema adjacent to physis } & Oedema not adjacent to physis \\
\hline Signal intensity ${ }^{\star}$ & No oedema & 2 & 4 & \\
\hline Visibility on 3D WATSc* & No oedema & \multicolumn{2}{|c|}{ Oedema not visible } & Oedema visible \\
\hline \multicolumn{5}{|l|}{ Physis } \\
\hline Thickness $^{920}$ & Normal & \multicolumn{2}{|l|}{ Increased } & \\
\hline Location of thickness ${ }^{20}$ & $\begin{array}{l}\text { No increased } \\
\text { thickness }\end{array}$ & \multicolumn{2}{|c|}{ Increased on radial side } & Increased on ulnar side \\
\hline $\begin{array}{l}\text { Thickness compared with proximal } \\
\text { physis of } \mathrm{MC}-1^{*}\end{array}$ & Not increased & Twice & Three times & Four times \\
\hline Signal intensity ${ }^{8}$ & 1 & 3 & 5 & \\
\hline Disruptions $^{32} 33$ & No disruptions & 1 & $>2$ disruptions & \\
\hline Width of disruptions ${ }^{*}$ & No disruptions & $<2 \mathrm{~mm}$ & $\geq 2 \mathrm{~mm}$ & \\
\hline Physeal border on epiphyseal side ${ }^{25}$ & Undulating & \multicolumn{2}{|c|}{ Irregular } & \\
\hline Depth of irregularities* & No irregularities & \multicolumn{2}{|c|}{$<$ Thickness of physis } & >Thickness of physis \\
\hline \multicolumn{5}{|l|}{ Metaphysis } \\
\hline Physeal border on metaphyseal side ${ }^{25}$ & Undulating & \multicolumn{2}{|c|}{ Slightly irregular } & Distinctly irregular \\
\hline Metaphyseal intrusions ${ }^{32} 34$ & Absent & \multicolumn{2}{|c|}{ Present } & \\
\hline Signal intensity intrusions ${ }^{*}$ & No intrusions & \multicolumn{2}{|c|}{$\begin{array}{l}\text { Less than } \\
\text { physis }\end{array}$} & Higher than physis \\
\hline Connection of intrusion with physis ${ }^{\star}$ & No intrusions & \multicolumn{2}{|c|}{ Connected with physis } & Not connected with physis \\
\hline Depth of intrusions ${ }^{*}$ & No intrusions & \multicolumn{2}{|l|}{$<2 \mathrm{~mm}$} & $>2 \mathrm{~mm}$ \\
\hline \multicolumn{5}{|l|}{ Bone marrow oedema } \\
\hline Presence ${ }^{1032}$ & Present & \multicolumn{2}{|l|}{ Absent } & \\
\hline Depth* & No oedema & \multicolumn{2}{|c|}{ Area $<2 \mathrm{~cm}$ from physis } & Area $\geq 2 \mathrm{~cm}$ from physis \\
\hline Location $^{1032}$ & No oedema & \multicolumn{2}{|c|}{ Oedema adjacent to physis } & Oedema not adjacent to physis \\
\hline Signal intensity* & No oedema & 12 & 4 & \\
\hline Visibility on 3D WATSc* & No oedema & \multicolumn{2}{|c|}{ Oedema not visible } & Oedema visible \\
\hline Homogeneity* & No oedema & \multicolumn{2}{|c|}{ Homogeneous oedema } & Inhomogeneous oedema \\
\hline Shape $^{25}$ & Normal & \multicolumn{2}{|c|}{ Unilateral widening } & Bilateral widening \\
\hline Location of widening* & No widening & \multicolumn{2}{|l|}{ Radial } & Ulnar \\
\hline Periosteal bone formation ${ }^{10}$ & Absent & \multicolumn{2}{|l|}{ Present } & \\
\hline Sclerosis $^{29}$ & Absent & \multicolumn{2}{|l|}{ Present } & \\
\hline
\end{tabular}

*Item added based on clinical experience with evaluation of physeal stress injuries.

3D WATSc, three-dimensional water-selective cartilage scan; MC-1, first metacarpal bone.

results show that gradient-echo images may be useful in identifying severe cases of periphyseal BMO.

\section{Physeal changes}

Increased physeal thickness is a frequently described sign of stress injury on conventional radiographs and MRI. ${ }^{2025}$ During normal growth, thickness remains constant almost until maturity, when the physis thins and eventually disappears. ${ }^{4}$ Physeal widening is thought to be caused by disrupted enchondral calcification, leading to accumulation of hypertrophied cartilage cells failing to ossify. ${ }^{2627}$
This pathological process can occur focally with unilateral widening or affect the entire physis (figure 3B). We propose a method to assess distal radial and ulnar physeal thickness by comparison with the proximal MC-1 physis. Since this bone suffers less repetitive axial loading than the radius and ulna in gymnasts, we used this as within-person 'reference physis' that can easily be included in the field of view.

Other studies have also described bony bridges indicating physeal closure, sometimes prematurely, after injury. ${ }^{28} 29$ D volumetric reconstruction of the physis 

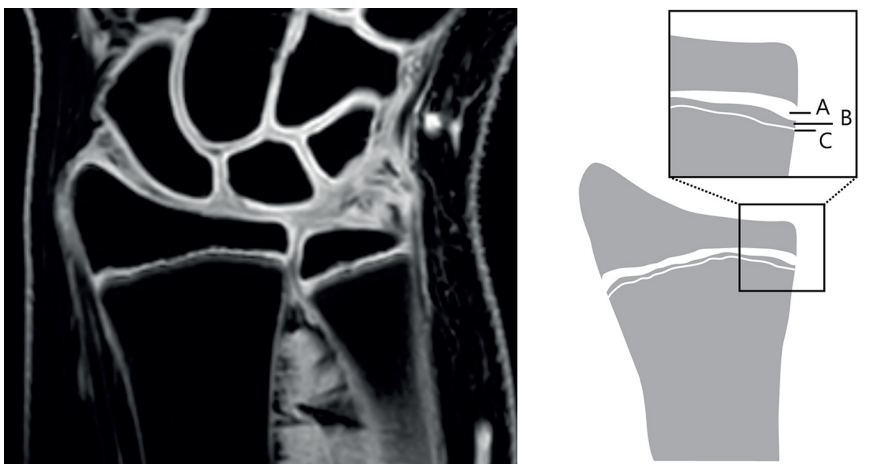

Figure 2 Left: three-dimensional water-selective cartilage scan image showing the trilaminar appearance of the physis. Right: schematic overview of the three layers of the physis. A: cartilaginous part, B: zone of provisional calcification, C: primary spongiosa of metaphysis.

and bony bars was proposed to aid in treatment decision-making. ${ }^{13}$ We found poor reliability for physeal disruptions on MRI, suggesting these characteristics are less suitable for physeal assessment. Interpretation possibly depends on disruption size: small physeal disruptions that were seen in all three participant groups were not graded as such by all observers. These may be signs of normal physeal maturation, or susceptibility artefacts associated with gradient-echo MRI, likely caused by calcium depositions during local growth plate closure.
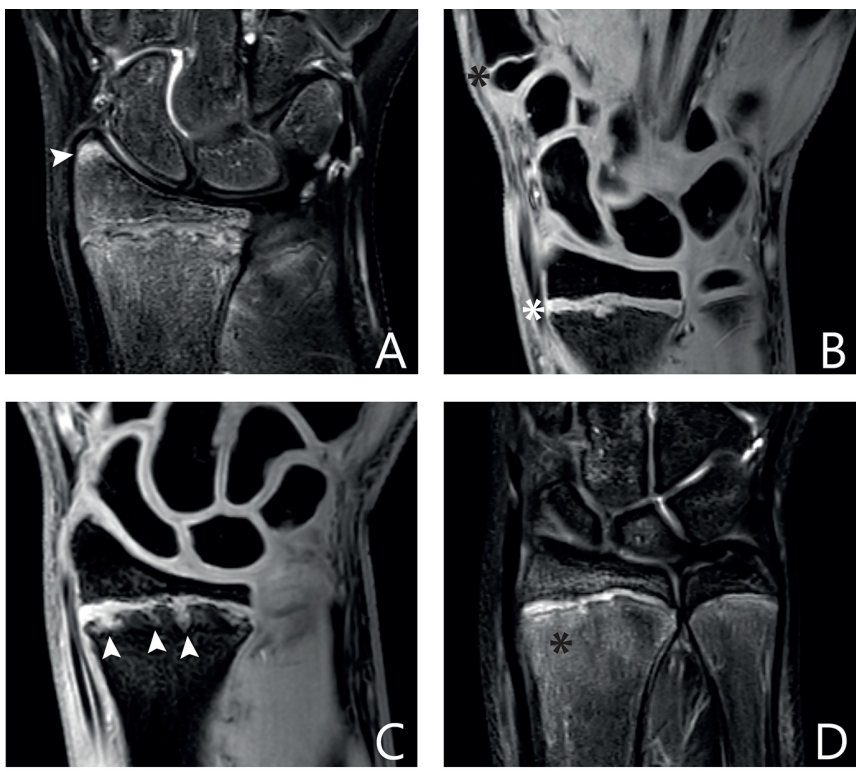

Figure 3 (A) T2 Dixon image showing a focal patch of epiphyseal bone marrow oedema in the radius, indicated by a white arrowhead. (B) Three-dimensional water-selective cartilage scan (3D WATSc) image showing diffuse thickening of the distal radial physis (located to the right of the white asterisk) compared with the proximal physis of the first metacarpal bone (located to the right of the black asterisk). (C) 3D WATSc image showing intrusions of physeal cartilage into the radial metaphysis, marked by white arrowheads.

(D) T2 Dixon image showing extensive bone marrow oedema of the radial metaphysis, marked with a black asterisk.
Physeal haziness or decreased radiolucency, another literature-derived characteristic of physeal stress injury, was excluded because it is mainly assessed on radiographs. ${ }^{25}$ Its MRI equivalent may be increased physeal signal intensity, reported after indirect physeal trauma. ${ }^{826}$ Although most of this study's MRI sequences depict the physis as a relatively high signal structure, physeal signal intensity showed fair reliability and can be indicative of injury.

\section{Metaphyseal changes}

The physeal appearance on MRI is trilaminar: a hyperintense cartilaginous layer, a hypointense zone of provisional calcification and a hyperintense region of primary spongiosa or metaphyseal vascularisation (figure 2). ${ }^{330}$ The healthy physis can appear undulated. ${ }^{5}$ Irregularity of physeal borders is described on radiographs and MRI of gymnasts with stress injury of the distal radial physis. ${ }^{25}$ Our results show better inter-rater agreement for irregularity of the metaphyseal border compared with the epiphyseal side. Discontinuations of the metaphyseal provisional calcification zone are known injury signs. ${ }^{14}$ Most likely, the physeal-metaphyseal junction is more frequently affected by stress injury because of its role in chondrocyte calcification during longitudinal growth (figure 2).

Similarly, intrusions or physeal cartilage 'tongues' with signal intensities similar to the physis can extend into the metaphysis after physeal injury (figure 3C). ${ }^{26} 27$ Reliability was moderate to almost perfect for the presence of metaphyseal intrusions in both the radius and the ulna. Physeal connection and depth of these intrusions showed fair to good inter-rater agreement, while intra-rater agreement was excellent. Thus, interpretation of a high signal area's physeal connection varies largely between observers and may in some cases include focal patches of high signal intensity also interpretable as metaphyseal BMO or FOPE. Intrusion extent or depth may therefore be more reliable injury signs.

Other metaphyseal changes associated with physeal stress injury include widening, cystic changes, sclerosis and striations on radiographs, and low signal and periosteal bone formation on MRI. ${ }^{9} 1026$ These were excluded from the AMPHYS protocol because of poor reliability, likely caused by low prevalence in all groups.

\section{Strengths and limitations}

The concise AMPHYS protocol provides reliable physeal assessment while minimising scan time, and accompanying patient burden. We aimed to achieve good validity by involving a varied group of five observers for reliability assessment, and a heterogeneous sample of symptomatic and asymptomatic gymnasts and non-gymnasts to ensure sufficient population variety. However, some variability in abnormalities between groups may be caused by artefacts interpreted as (peri)physeal changes, like susceptibility artefacts on 3D WATSc and chemical shift artefacts on 
Table 2 Intra-rater and inter-rater agreement for items relating to the radius and ulna

\begin{tabular}{|c|c|c|c|c|c|c|c|c|}
\hline \multirow[b]{3}{*}{ Categories and items } & \multicolumn{4}{|c|}{ Radius } & \multicolumn{4}{|l|}{ Ulna } \\
\hline & \multicolumn{2}{|c|}{$\begin{array}{l}\text { Intra-rater } \\
\text { agreement }\end{array}$} & \multicolumn{2}{|c|}{ Inter-rater agreement } & \multicolumn{2}{|c|}{ Intra-rater agreement } & \multicolumn{2}{|c|}{ Inter-rater agreement } \\
\hline & ICC & $95 \% \mathrm{Cl}$ & ICC & $95 \% \mathrm{Cl}$ & ICC & $95 \% \mathrm{Cl}$ & ICC & $95 \% \mathrm{Cl}$ \\
\hline \multicolumn{9}{|l|}{ Epiphysis } \\
\hline \multicolumn{9}{|l|}{ Bone marrow oedema } \\
\hline Extent & 0.86 & 0.72 to 0.94 & 0.78 & 0.66 to 0.88 & 0.74 & 0.53 to 0.87 & 0.30 & 0.15 to 0.50 \\
\hline Location & 0.60 & 0.32 to 0.79 & 0.60 & 0.43 to 0.75 & 0.69 & 0.44 to 0.84 & 0.26 & 0.11 to 0.45 \\
\hline Signal intensity & 0.85 & 0.69 to 0.93 & 0.76 & 0.63 to 0.86 & 0.59 & 0.30 to 0.78 & 0.27 & 0.12 to 0.47 \\
\hline Visibility on 3D WATSc & 0.90 & 0.79 to 0.95 & 0.70 & 0.55 to 0.83 & 0.28 & 0 to 0.57 & 0.34 & 0.18 to 0.54 \\
\hline \multicolumn{9}{|l|}{ Physis } \\
\hline Thickness* & 0.80 & 0.62 to 0.90 & 0.47 & 0.35 to 0.59 & 0.44 & 0.09 to 0.69 & 0.22 & 0.10 to 0.34 \\
\hline Location of thickness & 0 & 0 to 0.45 & 0.07 & 0 to 0.24 & 0 & NA & 0 & 0 to 0.14 \\
\hline $\begin{array}{l}\text { Thickness compared with proximal } \\
\text { physis of MC-1 }\end{array}$ & 0.83 & 0.67 to 0.91 & 0.58 & 0.42 to 0.73 & 0.52 & 0.19 to 0.74 & 0.21 & 0.07 to 0.40 \\
\hline Signal intensity & 0.81 & 0.65 to 0.90 & 0.62 & 0.47 to 0.76 & 0.62 & 0.35 to 0.80 & 0.39 & 0.23 to 0.58 \\
\hline Disruptions & 0.36 & 0 to 0.64 & 0.23 & 0.08 to 0.43 & 0.23 & 0 to 0.52 & 0.25 & 0.10 to 0.45 \\
\hline Width of disruptions & 0.39 & 0.02 to 0.66 & 0.26 & 0.11 to 0.45 & 0.42 & 0.09 to 0.67 & 0.26 & 0.11 to 0.46 \\
\hline Physeal border on epiphyseal side* & 0.67 & 0.40 to 0.83 & 0.35 & 0.23 to 0.47 & 0.67 & 0.42 to 0.83 & 0.20 & 0.08 to 0.32 \\
\hline Depth of irregularities & 0 & 0 to 0.36 & 0 & 0 to 0.15 & 0 & 0 to 0.36 & & 0 to 0.13 \\
\hline \multicolumn{9}{|l|}{ Metaphysis } \\
\hline Physeal border on metaphyseal side & 0.84 & 0.69 to 0.92 & 0.60 & 0.43 to 0.75 & 0.58 & 0.28 to 0.78 & 0.43 & 0.27 to 0.61 \\
\hline Metaphyseal intrusions ${ }^{\star}$ & 0.85 & 0.71 to 0.93 & 0.59 & 0.47 to 0.71 & 0.71 & 0.46 to 0.86 & 0.57 & 0.44 to 0.69 \\
\hline Signal intensity intrusions & 0.75 & 0.53 to 0.87 & 0 & 0 to 0.15 & 0.73 & 0.46 to 0.87 & 0 & 0 to 0.15 \\
\hline Connection of intrusion with physis & 0.85 & 0.71 to 0.93 & 0.41 & 0.24 to 0.60 & 0.56 & 0.25 to 0.77 & 0.43 & 0.26 to 0.62 \\
\hline Depth of intrusions & 0.91 & 0.82 to 0.96 & 0.69 & 0.54 to 0.82 & 0.67 & 0.36 to 0.84 & 0.54 & 0.38 to 0.71 \\
\hline \multicolumn{9}{|l|}{ Bone marrow oedema } \\
\hline Presence* $^{*}$ & 0 & 0 to 0.36 & 0.32 & 0.21 to 0.44 & 0 & NA & 0.33 & 0.21 to 0.45 \\
\hline Depth & 0 & 0 to 0.36 & 0.36 & 0.20 to 0.56 & 0 & NA & 0.28 & 0.12 to 0.49 \\
\hline Location & 0 & NA & 0.34 & 0.18 to 0.53 & 0 & NA & 0.34 & 0.18 to 0.54 \\
\hline Signal intensity & 0.55 & 0.22 to 0.76 & 0.51 & 0.35 to 0.69 & 0.46 & 0.10 to 0.71 & 0.43 & 0.26 to 0.61 \\
\hline Visibility on 3D WATSc & 0.43 & 0.11 to 0.68 & 0.31 & 0.16 to 0.51 & 0.23 & 0 to 0.52 & 0.34 & 0.18 to 0.53 \\
\hline Homogeneity & 0 & 0 to 0.04 & 0.34 & 0.14 to 0.58 & 0 & 0 to 0.02 & 0.15 & 0.03 to 0.34 \\
\hline Shape & 0 & 0 to 0.36 & 0.33 & 0.17 to 0.52 & 0 & 0 to 0.34 & 0.12 & 0.01 to 0.28 \\
\hline Location of widening & 0 & NA & 0.10 & 0 to 0.28 & 0 & 0 to 0.35 & 0 & 0 to 0.14 \\
\hline Periosteal bone formation* & 0 & NA & 0.26 & 0.14 to 0.39 & 0 & NA & 0 & 0 to 0.09 \\
\hline Sclerosis* & 0.23 & 0 to 0.54 & 0.08 & 0 to 0.22 & 0 & NA & 0 & 0 to 0.04 \\
\hline
\end{tabular}

*Fleiss' kappa is reported for dichotomous variables.

3D WATSc, three-dimensional water-selective cartilage scan; ICC, intraclass correlation coefficient; MC-1, first metacarpal bone; NA, not available (due to zero variance).

spin-echo PD images. In addition, the MC-1 physis is less reliable as reference for thickness when it has nearly fused. Nevertheless, assessment of radial physeal thickness by itself also showed moderate to substantial inter-rater and intra-rater agreement. Finally, sample size was based on the aim to assess the protocol's reliability. For assessment of its diagnostic accuracy and score interpretation, a separate study in a larger sample is necessary. ${ }^{1731}$

\section{Clinical implications and future directions}

The patient-friendly and radiologist-friendly AMPHYS protocol is directly available for standardised and quick assessment of periphyseal changes in children with suspected physeal injury. MRI of the wrist on 3T is currently the standard of care in many clinical settings, and the prevalent sequences on which the evaluation protocol is based are supplied by most vendors and can likely even be 


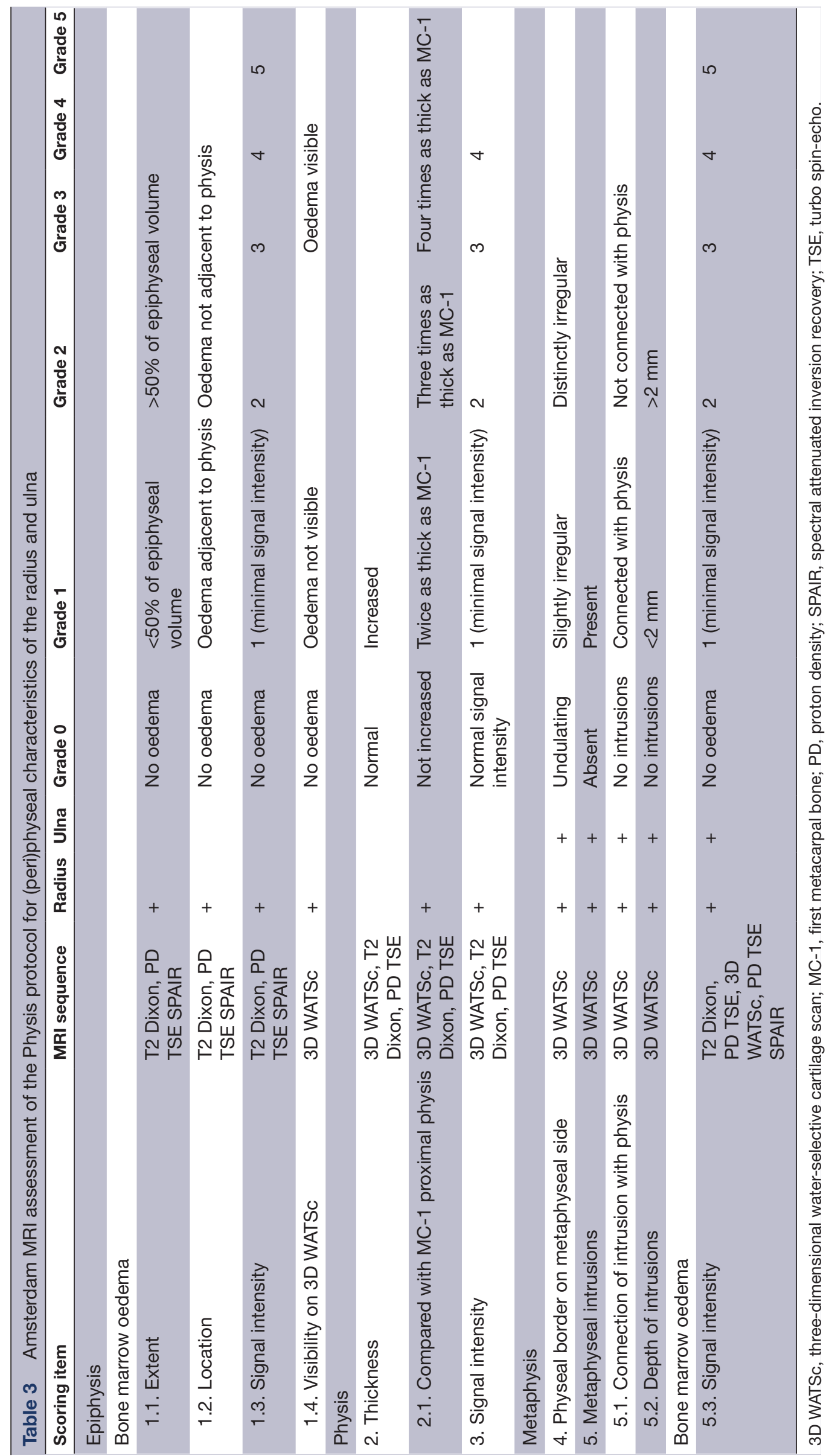


modified for $1.5 \mathrm{~T}$ scanners. In addition, patient burden is minimal, with absence of ionising radiation and a scan time of less than $15 \mathrm{~min}$. The protocol can be used for initial injury assessment, treatment and recovery evaluation, and monitoring of (peri) physeal changes in children at risk of physeal injury. Uniform reporting of physeal stress changes on MRI can contribute to patient care and further research on related topics such as prognosis of injury recovery and potential complications.

This study provides data from gymnasts as the patient group most frequently affected with physeal stress injury of the wrist. Comparison with asymptomatic gymnasts is recommended because of changes that can be present due to physiological responses to exercise. Outcome scores need to be validated on a larger scale to provide diagnostic accuracy, grading interpretation and cut-off values for presence and severity of physeal stress injury. We will proceed to evaluate this grading system in a larger cohort study.

\section{CONCLUSION}

The AMPHYS protocol is a concise collection of radiographic and MRI-based characteristics of the periphyseal area of the radius and ulna that can be reliably assessed on 3T MRI after merely 15 minutes of scan time. Its 12 items include epiphyseal and metaphyseal BMO, physeal thickness and signal intensity, and metaphyseal intrusions and irregularities, with fair to excellent inter-rater and intra-rater agreement.

\author{
Author affiliations \\ ${ }^{1}$ Department of Radiology and Nuclear Medicine, Academic Medical Center \\ Amsterdam, The Netherlands \\ ${ }^{2}$ Academic Center for Evidence-based Sports medicine (ACES) Amsterdam, The \\ Netherlands \\ ${ }^{3}$ Amsterdam Collaboration for Health and Safety in Sports (ACHSS), International \\ Olympic Committee (IOC) Research Center AMC/NUmc Amsterdam, The Netherlands \\ ${ }^{4}$ Department of Radiology and Nuclear Medicine, Noordwest Hospital Group \\ Alkmaar, The Netherlands \\ ${ }^{5}$ Department of Radiology, St. Antonius Hospital Utrecht, The Netherlands \\ ${ }^{6}$ Department of Radiology and Nuclear Medicine, Erasmus MC Rotterdam, The \\ Netherlands \\ ${ }^{7}$ Department of Radiology, Amphia Hospital Breda, The Netherlands
}

Twitter Presenting the concise AMPHYS protocol: characteristics of the growth plate area of the radius and ulna that can be reliably assessed on 3T MRI after merely 15 minutes of scan time.

Acknowledgements The authors thank the athletes for their contribution to the study, and Valentina Mazzoli, PhD, Jos Oudeman, MD, PhD, Aart Nederveen, PhD, and Marieke Biegstraaten, MD, PhD, for their assistance in setting up the study's methodology and Sandra van den Berg-Faay for her assistance in performing the MRI scans. The research was conducted as part of the Sports \& Work research programme of Amsterdam Movement Sciences.

Contributors LSK, RBJK and MM conceived of the study and participated in its design. LSK coordinated the study. MCdJ, EHGO and MM took part in the expert group in the development phase, and KFvD, RH, SJ, FFS and MPT participated in the expert group in the validation phase. LSK and RBJK carried out the data collection and performed the data analysis. All authors provided input for the manuscript, which was drafted by LSK. All authors commented on the draft versions of the manuscript and have read and approved its final version.

Funding This work was supported by the Academic Medical Center, Amsterdam, The Netherlands, under an AMC PhD Scholarship 2013, awarded to the author LSK. Competing interests None declared.
Ethics approval Approval was granted from the Medical Ethics Commission of the Academic Medical Center.

Provenance and peer review Not commissioned; internally peer reviewed.

Open Access This is an Open Access article distributed in accordance with the Creative Commons Attribution Non Commercial (CC BY-NC 4.0) license, which permits others to distribute, remix, adapt, build upon this work non-commercially, and license their derivative works on different terms, provided the original work is properly cited and the use is non-commercial. See: http://creativecommons.org/ licenses/by-nc/4.0/

(c) Article author(s) (or their employer(s) unless otherwise stated in the text of the article) 2018. All rights reserved. No commercial use is permitted unless otherwise expressly granted.

\section{REFERENCES}

1. Jaimes C, Chauvin NA, Delgado J, et al. MR imaging of normal epiphyseal development and common epiphyseal disorders. Radiographics 2014;34:449-71.

2. Davis KW. Imaging pediatric sports injuries: upper extremity. Radiol Clin North Am 2010;48:1199-211.

3. Jaramillo D, Connolly SA, Mulkern RV, et al. Developing epiphysis: MR imaging characteristics and histologic correlation in the newborn lamb. Radiology 1998;207:637-45.

4. Laor T, Jaramillo D. MR imaging insights into skeletal maturation: What is normal? Radiology 2009;250:28-38.

5. Ecklund K, Jaramillo D. Imaging of growth disturbance in children. Radiol Clin North Am 2001;39:823-41.

6. Kox LS, Kuijer PP, Kerkhoffs GM, et al. Prevalence, incidence and risk factors for overuse injuries of the wrist in young athletes: a systematic review. Br J Sports Med 2015;49:1189-96.

7. Jaimes $C$, Jimenez M, Shabshin N, et al. Taking the stress out of evaluating stress injuries in children. Radiographics 2012;32:537-55.

8. Delgado J, Jaramillo D, Chauvin NA. Imaging the injured pediatric athlete: upper extremity. Radiographics 2016;36:1672-87.

9. DiFiori JP, Puffer JC, Aish B, et al. Wrist pain, distal radial physeal injury, and ulnar variance in young gymnasts: does a relationship exist? Am J Sports Med 2002;30:879-85.

10. Jawetz ST, Shah PH, Potter HG. Imaging of physeal injury: overuse. Sports Health 2015;7:142-53.

11. Disler DG. Fat-suppressed three-dimensional spoiled gradientrecalled MR imaging: assessment of articular and physeal hyaline cartilage. AJR Am J Roentgenol 1997;169:1117-23.

12. Maas M, Dijkstra PF, Akkerman EM. Uniform fat suppression in hands and feet through the use of two-point Dixon chemical shift MR imaging. Radiology 1999;210:189-93.

13. Lurie B, Koff MF, Shah P, et al. Three-dimensional magnetic resonance imaging of physeal injury: reliability and clinical utility. $\checkmark$ Pediatr Orthop 2014;34:239-45.

14. Avenarius DFM, Ording Müller LS, Rosendahl K. Joint fluid, bone marrow edemalike changes, and ganglion cysts in the pediatric wrist: features that may mimic pathologic abnormalities-follow-up of a healthy cohort. AJR Am J Roentgenol 2017;208:1352-7.

15. Müller LS, Avenarius D, Damasio B, et al. The paediatric wrist revisited: redefining MR findings in healthy children. Ann Rheum Dis 2011;70:605-10.

16. van Rijn RR, Lequin $\mathrm{MH}$, Thodberg $\mathrm{HH}$. Automatic determination of Greulich and Pyle bone age in healthy Dutch children. Pediatr Radiol 2009;39:591-7.

17. De Vet HC, Terwee CB, Mokkink LB, et al. Measurement in medicine: a practical guide: Cambridge University Press, 2011.

18. Cicchetti DV. Guidelines, criteria, and rules of thumb for evaluating normed and standardized assessment instruments in psychology. Psychol Assess 1994;6:284-90.

19. Landis JR, Koch GG. The measurement of observer agreement for categorical data. Biometrics 1977;33:159-74.

20. Roy S, Caine D, Singer KM. Stress changes of the distal radial epiphysis in young gymnasts. A report of twenty-one cases and a review of the literature. Am J Sports Med 1985;13:301-8.

21. Zbojniewicz AM, Laor T, Edema FP. Focal Periphyseal Edema (FOPE) zone on MRI of the adolescent knee: a potentially painful manifestation of physiologic physeal fusion? AJR Am J Roentgenol 2011;197:998-1004.

22. Grampp S, Henk CB, Mostbeck GH. Overuse edema in the bone marrow of the hand: demonstration with MRI. J Comput Assist Tomogr 1998;22:25-7.

23. Major NM, Helms CA. MR imaging of the knee: findings in asymptomatic collegiate basketball players. AJR Am J Roentgenol 2002;179:641-4. 
24. Peterfy CG, Gold G, Eckstein F, et al. MRI protocols for whole-organ assessment of the knee in osteoarthritis. Osteoarthritis Cartilage 2006;14(Suppl A):95-111.

25. DiFiori JP. Overuse injury and the young athlete: the case of chronic wrist pain in gymnasts. Curr Sports Med Rep 2006;5:165-7.

26. Jaramillo D, Laor T, Zaleske DJ. Indirect trauma to the growth plate: results of MR imaging after epiphyseal and metaphyseal injury in rabbits. Radiology 1993;187:171-8.

27. Laor T, Wall EJ, Vu LP. Physeal widening in the knee due to stress injury in child athletes. AJR Am J Roentgenol 2006;186:1260-4.

28. Ecklund $\mathrm{K}$, Jaramillo $\mathrm{D}$. Patterns of premature physeal arrest: MR imaging of 111 children. AJR Am J Roentgenol 2002;178:967-72.

29. Ogden JA. The evaluation and treatment of partial physeal arrest. J Bone Joint Surg Am 1987;69:1297-302.
30. Jaramillo D, Shapiro F. Growth cartilage: normal appearance, variants and abnormalities. Magn Reson Imaging Clin N Am 1998;6:455-71.

31. Bossuyt PM, Reitsma JB, Bruns DE, et al. STARD Group. STARD 2015: an updated list of essential items for reporting diagnostic accuracy studies. Radiology 2015;277:826-32.

32. Paz DA, Chang GH, Yetto JM, et al. Upper extremity overuse injuries in pediatric athletes: clinical presentation, imaging findings, and treatment. Clin Imaging 2015;39:954-64.

33. Lomasney LM, Lim-Dunham JE, Cappello T, et al. Imaging of the pediatric athlete: use and overuse. Radiol Clin North Am 2013;51:215-26.

34. Dwek JR, Cardoso F, Chung CB. MR imaging of overuse injuries in the skeletally immature gymnast: spectrum of soft-tissue and osseous lesions in the hand and wrist. Pediatr Radiol 2009;39:1310-6. 